\title{
AGO Recommendations for Diagnosis and Treatment of Patients with Primary and Metastatic Breast Cancer. Update 2011
}

\author{
Christoph Thomssen ${ }^{\mathrm{a}}$ Anton Scharl ${ }^{\mathrm{b}}$ Nadia Harbeck $^{\mathrm{c}}$, on behalf of the AGO Breast Committee* \\ ${ }^{a}$ Frauenklinik, Martin Luther Universität Halle (Saale), \\ ${ }^{\mathrm{b}}$ Frauenklinik, Klinikum Amberg, \\ 'Brustzentrum, Unifrauenklinik Köln, Germany
}

\section{Introduction}

For the last 10 years, the Breast Committee of the Arbeitsgemeinschaft Gynäkologische Onkologie (German Gynecological Oncology Group, AGO) has been preparing and updating evidence-based recommendations for the diagnosis and treatment of patients with early breast cancer and metastatic breast cancer (MBC). The AGO Breast Committee consists of 44 gynecological oncologists specialized in breast cancer as well as interdisciplinary members specialized in pathology, radiological diagnostics, medical oncology, and radiotherapy. The update is performed according to documented rules by thoroughly reviewing and scoring chapter by chapter the recent publications for its scientific validity (Oxford Level of Evidence; LoE; www.cebm.net [34]) and its clinical relevance (AGO Grades of Recommendation; table 1). Here, we present the 2011 update of these guidelines focusing on modifications performed for the 2011 version. The full version of the update is available in PDF format

\footnotetext{
*Members of the 'AGO Breast Committee' in alphabetical order: Ute-Susan Albert, Marburg; Ingo Bauerfeind, Landshut; Joachim Bischoff, Magdeburg; Jens Uwe Blohmer, Berlin; Klaus Brunnert, Osnabrück; Peter Dall, Lüneburg; Ingo J. Diel, Mannheim; Tanja Fehm, Tübingen; Nikos Fersis, Chemnitz; Michael Friedrich, Krefeld; Kay Friedrichs, Hamburg; Bernd Gerber, Rostock; Volker Hanf, Fürth; Nadia Harbeck, Köln; Jens Huober, St. Gallen; Christian Jackisch, Offenbach; Wolfgang Janni, Düsseldorf (Co-Chair); Walter Jonat, Kiel (DKH); Hans H. Kreipe, Hannover (DGP); Sherko Kümmel, Essen; Sibylle Loibl, Neu-Isenburg; Hans-Joachim Lück, Hannover; Michael Lux Erlangen; Nicolai Maass, Aachen; Gunter von Minckwitz, Neu-Isenburg; Volker Möbus, Frankfurt; Christoph Mundhenke, Kiel; Volkmar Müller, Hamburg; Ulrike Nitz, Mönchengladbach; Mahdi Rezai, Düsseldorf; Achim Rody, Frankfurt; Anton Scharl, Amberg (Chair); Rita Schmutzler, Köln; Marcus Schmidt, Mainz; Andreas Schneeweiß, Heidelberg (AIO); Ingrid Schreer, Kiel (DGS); Florian Schütz, Heidelberg; Peter Sinn, Heidelberg (Pathologie); Erich F. Solomayer, Homburg; Rainer Souchon, Tübingen (ARO); Elmar Stickeler, Freiburg; Christoph Thomssen, Halle (Saale); Michael Untch, Berlin
}

online (www.ago-online.de, 'Leitlinien') [2] in an English and a German version.

\section{Adjuvant Endocrine Therapy \\ in Premenopausal Patients}

Regarding adjuvant endocrine therapy for premenopausal patients with endocrine sensitive disease, the AGO recommendations have not changed substantially in 2011. Assessment of the menopausal status is best done by menstruation history and follicle-stimulating hormone (FSH) and estradiol (E2) measurements. Low anti-muellerian hormone (AMH) levels seem to be indicative for reduced ovarian reserve and chemotherapy-related amenorrhea (CRA) in chemotherapytreated breast cancer patients $[177,213]$. AMH measurements may thus help to assess the ovarian reserve after chemotherapy (AGO +/-).

Tamoxifen alone is the preferred endocrine therapy in young women (AGO ++; EBCTCG 2005), in cases of tamoxifen contraindications, gonadotropin-releasing hormone analogues (GnRHa) alone may be an alternative in patients with low (and intermediate) risk of recurrence as the long-term results of the ZIPP trial suggest [99]. Due to the side effect profile and remaining uncertainties when looking at the individual (and overall low) event number in the ABCSG 12 trial, GnRHa and aromatase inhibitors (AI) are not considered a regular therapeutic option in premenopausal patients with endocrine sensitive disease (AGO -). Yet, the experts feel that GnRHa and AI may be considered in patients with severe contraindications against tamoxifen (AGO $+/-$ ). Based on the convincing data from MA17 [96], extended adjuvant therapy with an AI is a valid option for premenopausal patients that have become postmenopausal during

\section{KARGER}

Fax +497614520714

Information@Karger.de

www.karger.com (c) 2011 S. Karger GmbH, Freiburg

1661-3791/11/0064-0299\$38.00/0

Accessible online at:

www.karger.com/brc
Prof. Dr. med. Christoph Thomssen

Klinik und Poliklinik für Gynäkologie

Martin-Luther-Universität Halle-Wittenberg

Ernst-Grube-Strasse 40, D-06097 Halle/Saale, Germany

Tel. +49 345 5571-847, Fax -501

christoph.thomssen@medizin.uni-halle.de 
Table 1. AGO grades of recommendation

\begin{tabular}{ll}
\hline++ & This investigation or therapeutic intervention is highly beneficial for patients, can be recommended without restriction, and should \\
be performed. \\
+ \\
This investigation or therapeutic intervention is of limited benefit for patients and can be performed. \\
+- \\
This investigation or therapeutic intervention has not shown benefit for patients and may be performed only in individual cases. \\
According to current knowledge a general recommendation cannot be given. \\
- This investigation or therapeutic intervention can be of disadvantage for patients and might not be performed. \\
This investigation or therapeutic intervention is of clear disadvantage for patients and should be avoided or omitted in any case.
\end{tabular}

their 5 years of tamoxifen $(\mathrm{AGO}+)$. Fertility protection is an important topic for counseling young breast cancer patients. Due to the heterogeneous evidence and the possibility of a reduced chemotherapy efficacy when simultaneous endocrine treatment is performed, GnRHa are not recommended for routine ovarian protection (AGO -). Useful information regarding all aspects of fertility preservation is provided by the FertiPROTEKT network (www.fertiprotekt.de).

\section{Adjuvant Endocrine Therapy in Postmenopausal Patients}

With current guidelines for receptor testing, all tumors with one or more percent of positive cells for estrogen receptor (ER) and/or progesterone receptor (PR) should be considered as hormone receptor positive and patients are therefore candidates for endocrine therapy $[63,102,178]$ The 3 possible current attempts in endocrine treatment of postmenopausal patients comprise of sequential therapy $(\mathrm{AGO}++)$, upfront $\mathrm{AI}(\mathrm{AGO}+)$, and treatment with tamoxifen 5 years $(\mathrm{AGO}+)$. The sequential approach is either tamoxifen for 2-3 years followed by AI 3-2 years (LoE 1aA) or AI 2-3 years followed by tamoxifen for 3-2 years and a total treatment of 5 years (LoE $1 b C$ ), with a preference for the latter approach in nodepositive disease (data available only for letrozole).

A retrospective analysis of the ATAC trial stated that the relative efficacy of anastrozole compared to tamoxifen is greater in normal weight postmenopausal women. However, recurrence rates were lower for anastrozole than tamoxifen for all body mass index (BMI) quintiles. The authors speculated that higher doses or more potent AI may be more effective in overweight women, but also noted that this requires independent confirmation $[95,195]$. In a retrospective analysis of the TEAM trial, more disease events were observed after 2.75 years in obese women using tamoxifen in comparison to obese exemestane users, whereas at 5 years these differences in disease recurrences disappeared in this specific subgroup of patients [196]. In conclusion, current data do not justify the preferential use of $1 \mathrm{AI}$ or dose modifications.

\section{Adjuvant Chemotherapy plus Trastuzumab}

Essentially, with regard to adjuvant chemotherapy, we found more study results establishing those of the last years than really new approaches. With regard to taxane use, recent results and meta-analyses suggest that with anthracycline combinations equi-efficacy to taxane combinations can be achieved only if dosages were enhanced to toxic ranges [14, $28,69,118]$. Superiority of taxanes was demonstrated only in comparison to weak control arms [14, 52, 167]. Optimized schedules of taxane combinations like 4 courses of doxorubicin and cyclophosphamide (AC) / epirubicin and cyclophosphamide (EC) followed by 4 courses of docetaxel q3w or 4 courses of $\mathrm{AC} / \mathrm{EC}$ followed by 12 courses of pacitaxel $\mathrm{q} 1 \mathrm{w}$ were shown to be highly effective with acceptable side effect rates [205]. Thus, taxane-containing regimens are now considered standard in most indications ( $\mathrm{LoE} 1 \mathrm{aA} ; \mathrm{AGO}++)$, and if not for being more effective, than at least for having no cross-reactivity with anthracyclines. This is also true in nodenegative breast cancer ( $\mathrm{LoE} 1 \mathrm{~b}$; $\mathrm{AGO}+$ ).

In addition, dose-dense chemotherapy is now considered an established option. The German version ddETC (doseescalated sequential epirubicin, paclitaxel and cyclophosphamide) has demonstrated high efficacy in high-risk breast cancer ( $>3$ tumor-infiltrated lymph nodes) with a substantial advantage over a Henderson like EC-paclitaxel q3w with regard to disease-free survival (DFS) and overall survival (OS). The efficacy of ddETC seems to be independent from biological characteristics such as grading, hormone receptor status, and HER2 expression [159]. However, the US version, ddAC ( $\mathrm{AC} \mathrm{q} 2 \mathrm{w}$ followed by paclitaxel $\mathrm{q} 2 \mathrm{w}$ ) is effective especially in node-positive hormone receptor-negative patients (LoE 1aA; AGO ++) [44, 115]. Accepted regimens are: 4 courses $\mathrm{AC}$ or EC followed by 12 courses of paclitaxel weekly (AC-Pac, EC-Pac) or 4 courses of docetaxel q3w (ACDoc, EC-Doc), respectively; docetaxel in combination with AC (DAC); 3 courses French FEC (5-fluorouracil (5FU)/epirubicin/cyclophosphamide) followed by 3 courses of docetaxel (FEC-Doc). For other compounds (capecitabine, gemcitabine) or combinations (E-cisplatin-5-FU), there are promising data $[120,242]$, however, to date they are not convincing enough to be recommended for routine use (AGO $+/-)$. 
Finally, evidence is accumulating at least from large retrospective analyses, that small (pT1a,b) node-negative but HER2-overexpressing tumors have a higher risk of distant recurrence (approximately $15 \%$ after 5 years) than small HER2-negative tumors, even higher than small triple-negative tumors. On the other hand, the trastuzumab effect with regard to relative reduction of risk of relapse has been demonstrated to be stable between the subgroups, such that a reasonable effect can be extrapolated also for small HER2overexpressing tumors. In high-risk small and node-negative HER2-overexpressing tumor, adjuvant trastuzumab therapy is recommended $(\mathrm{LoE} 4 \mathrm{C}, \mathrm{AGO}+)$ [94, 223]. There is preference for administration of trastuzumab simultaneously at least to the taxane phase of adjuvant chemotherapy [181].

\section{Neoadjuvant (Primary) Systemic Therapy}

The main reason for primary or neoadjuvant chemotherapy is an increasing focus on in-situ-chemosensitivity testing instead of optimization of breast-conserving therapy. Therefore we included further parameters as predictive factors for achieving a pathological complete remission (pCR): Ki-67, topo-II- $\alpha$, and PARP (LoE $2 \mathrm{bB}, \mathrm{AGO}+/-$; participation in studies recommended).

Based on a German meta-analysis, a combination treatment with anthracyclines and taxanes lasting for more than 18 week carries the highest chance for pCR. Capecitabine may be added in locally advanced and inflammatory disease to optimize pCR rates (LoE 2bB, AGO +/-) [119, 146, 239, 241]. Platinum-containing therapies have shown efficacy in phase II trials in triple-negative disease [36, 249] and especially in BRCA-associated tumors [29]. The AGO Breast Committee stated that this treatment may be an individual option for some patients with triple-negative or BRCA-associated breast cancer (LoE $2 \mathrm{bB}, \mathrm{AGO}+/-$ ). It should be offered in clinical trials.

In HER2-overexpressing tumors, trastuzumab should be given routinely in combination with chemotherapies to improve pCR, DFS, and OS [84, 224] (1a, A, ++). The GeparQuinto trial has shown that lapatinib is not as effective as trastuzumab (LoE 2baB; AGO -). However, lapatinib as well as pertuzumab (not available in Europe outside clinical trials) can improve pCR rate if given additionally to trastuzumab and chemotherapy $[12,85,225]$. Especially lapatinib increases toxicities significantly without showing improvement in OS up to now. Therefore we do not recommend this dual anti$\mathrm{HER} / 2$ strategy routinely ( $\mathrm{LoE} 2 \mathrm{baB} ; \mathrm{AGO}+/-$ ).

Bevacizumab has not shown any effect on $\mathrm{pCR}$ rate in the German GeparQuinto trial and can not be recommended routinely (LoE 2bB, AGO -). However, in a subgroup analysis, bevacizumab induced higher $\mathrm{pCR}$ rates in triple-negative tumors. Thus this tumor type might be an indication for this agent [238]. Further trials should address this finding before it can be used in patients with triple-negative disease (LoE 2baC; $\mathrm{AGO}+/-$ ).

Neoadjuvant endocrine therapy is an option in patients with inoperable hormone receptor-positive disease, who deny chemotherapy (LoE 2bB; AGO +). AI should be given over a period of at least 3 months (LoE 1bB; $\mathrm{AGO}+$ ).

\section{Breast Cancer Surgery - Oncologic Aspects}

One of the most essential changes in the AGO Guidelines 2011 is the option (AGO +/-) of forgoing a subsequent axillary lymphadenectomy in patients with no more than 2 positive lymph nodes [127]. The basis for this change is the Z0011 study [87, 88]. However, the option of omitting an axillary dissection depends on the following conditions all of which have to be fulfilled: $\leq 2$ positive sentinel lymph nodes (macrometastases, no capsular penetration), breast-conserving surgery with cT1/2 tumors with subsequent adjuvant tangential radiation and adjuvant systemic therapy (anti-hormonal and/ or chemotherapeutic). However, the implementation of this recommendation into everyday clinical practice is difficult since it may detain relevant information for treatment decisions: Lack of knowledge concerning the exact number of affected lymph nodes might impede identification of patients with $\geq 4$ diseased lymph nodes, who will therefore not receive a potentially beneficial dose-intensified chemotherapy [44, 159]. Though it must be noted that the benefits of dose-dense and dose-intensified therapy have only been shown in patients who are $\leq 65$ years old [159].

Awareness of this clinical problem, which shows from the data of the study, was one of the reasons for the commission to give the recommendation $\mathrm{AGO}+/-$. The reservations reflect their judgment with respect to certain indications where the nodal status does not allow clear conclusions to be drawn. In situations outlined above, abstinence from axillary dissection should not result in irradiation of the axillary region. Preoperative diagnostics by means of ultrasound [25] (including axillary fine needle or core biopsy if necessary [2, 37, 114]) and knowledge of tumor biology and prognostic factors are of great significance for decisions on the surgical strategy in cases with positive sentinal lymph nodes. Nomograms can be of help [19, 232, 233].

The indications for sentinel lymph node excision (SLNE) were expanded by including T3 tumors $(+)$ since reliability of SLNE was shown also for larger tumors [127]. SLNE is optional in high-grade ductal carcinoma in situ (DCIS) $\geq 2.5 \mathrm{~cm}$ or in cases of mastectomy for DCIS $(\mathrm{AGO}+/-)$ since this poses a risk of upgrading to invasive disease.

\section{Plastic Reconstructive Aspects after Mastectomy}

For changes, see www.ago-online.de. 


\section{Lesions of Uncertain Malignant Potential (B3) and Pathology}

The guidelines for precursor lesions and pathology have been modified in some items which will be described below.

Requirement of Surgical Resection after Diagnosis of Atypical Ductal Hyperplasia in Core Biopsy

Atypical ductal hyperplasia (ADH) represents an intraductal proliferation of atypical epithelium which fulfils the criteria for low grade intraductal carcinoma (DCIS) but is restricted to 1 completely occupied duct or is no more than $2 \mathrm{~mm}$ in diameter. Because of this definition it seems to be impossible to differentiate $\mathrm{ADH}$ from low grade DCIS in core biopsies. Therefore, the recommendation of the AGO has been to perform complete resection of the suspicious area after a core biopsy diagnosis of ADH. Recently, some retrospective studies have been published which found a minimal risk of progression when the ADH lesion showed the following criteria $[3,165,180]$ : i) restriction to 2 or less terminal ductulo-lobular units; ii) no necrosis; iii) only moderate nuclear atypia.

Regarding complete removal of the suspicious area in the mammogram by vacuum biopsy, the AGO recommendations have been adapted accordingly. In ADH cases fulfilling the above mentioned criteria, resection can be avoided.

\section{Definition of Estrogen Receptor Positivity}

The method of determining the ER status is immunohistochemistry, polymerase chain reaction (PCR)-based methods are not recommended by the AGO. There are different definitions of thresholds which define a mammary cancer as being ER-positive (table 2). The AGO adapted the ASCO/CAP guidelines. Every report on ER expression should mention the percentage of positively stained tumor cells, scores can be provided but are not sufficient on their own.

\section{Triple-Negative Breast Cancer}

Triple-negative and basal breast cancer have been recognized as overlapping subtypes of breast cancer with a particular biological behavior [78]. The AGO prefers triple-negative instead of basal type of breast cancer. The latter provides a molecularly defined term which is still not translated into immunohistochemical definitions. Triple-negative breast cancer according to AGO criteria should exhibit the following features: ER < 1\%, PR < 1\%, HER2 0/1+2+ (non-amplified, ratio $\leq 2)$, not belonging to tumors of the salivary gland type (myoepithelial or adenoid-cystic carcinoma).

\section{Ki-67 Assessment}

The determination of the proliferative activity by Ki-67 immunohistochemistry has been proposed as a useful method to discriminate luminal A from luminal B type of breast cancer [92]. The AGO considers Ki-67 as a useful method to objectify histopathological grading, in particular in core biopsies, but does not recommend that every case should has to be labeled with Ki-67. The reasons for a '+/-'-recommendation by AGO is chiefly a lack of standardization: i) different antibodies and modes of antigen retrieval; ii) non-standardized method of assessment (random or non-random selection of microscopic fields, semiquantitative estimation or counting, number of cells to be counted, clear-cut criteria to discriminate positive from negative nuclear labeling); iii) lack of homogeneous figures in the literature to discriminate between low $(\leq 10-14 \%)$, intermediate, and high ( $\geq 25-30 \%)$ Ki-67 labeling index.

Table 2. Different definitions of estrogen receptor positivity in breast cancer

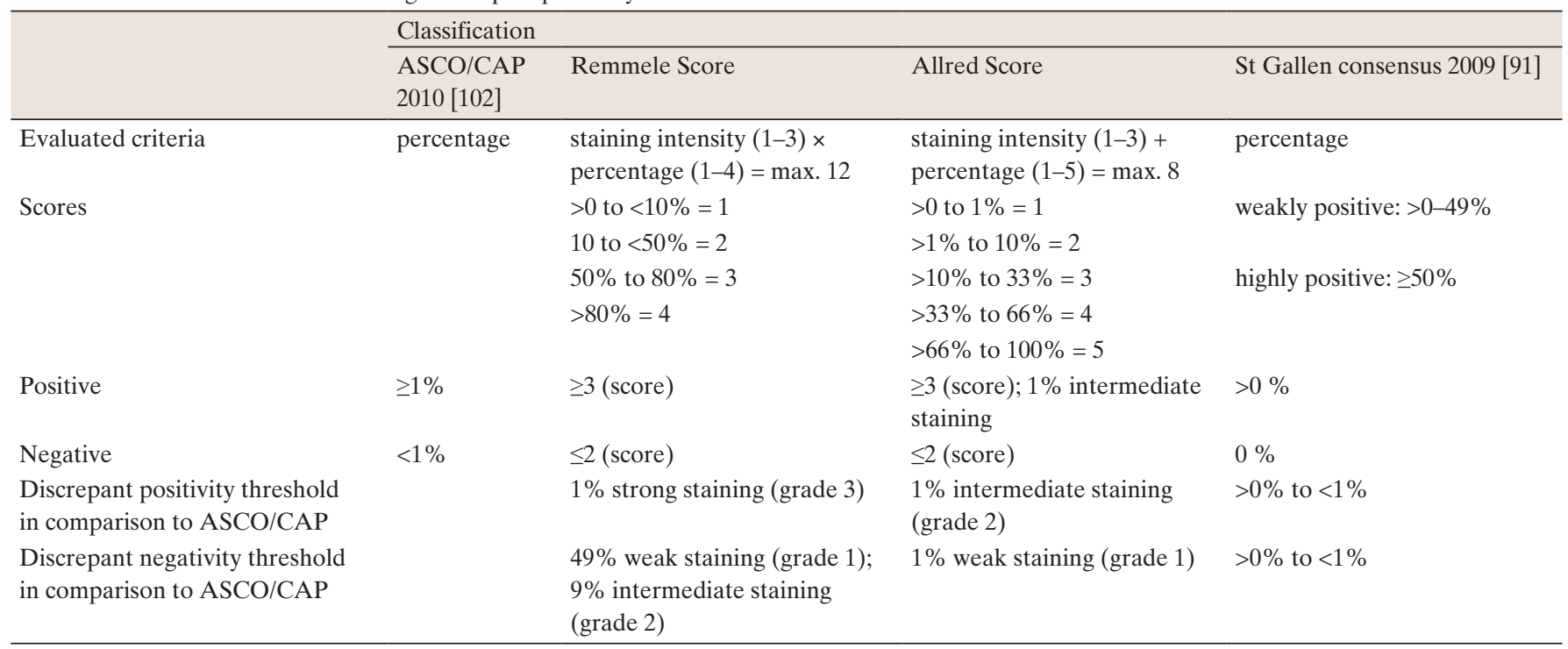




\section{Ductal Carcinoma in Situ}

DCIS make up for $20-25 \%$ of German screening cases. As for invasive ductal carcinoma the incidence has been falling since the late 1990ies. Most cases are diagnosed by the typical microcalcifications on mammography; additional magnetic resonance imaging (MRI) in most series improves DCIS detection (especially lesions not associated to microcalcifications) and improves lesion size determination. Preoperatively those patients with elevated risk of coincident invasive carcinoma, who benefit from sentinel lymph node biopsy (SLNB), are identified most accurately on the basis of grading and lesion size. Models including age, morphology, level of suspicion etc. modestly improve identification of this subset. Molecular profiling of DCIS demonstrates a wide biological heterogeneity without giving additional clinical information as known e.g. for invasive breast cancer.

Surgery is the main treatment option and should aim at complete resection of DCIS with free margins $>2 \mathrm{~mm}$. Prognostic factors for (non)-invasive recurrence are resection margins, microcalcifications, lesion size, grading, comedo necrosis, architecture, age, van Nuys prognostic index, radiotherapy, tamoxifen use, palpability, and immunohistochemistry (COX-2+, p16+, Ki-67+). Again no clinically relevant molecular profiling is available yet. However, on the basis of these prognosticators it is not possible to identify a subpopulation that can be spared postoperative radiotherapy as a recent phase III trial from EORTC demonstrated. Recently published large trials further support tamoxifen use in ERpositive disease.

In summary, DCIS is a locoregional disease, and treatment especially beyond surgery prevents (non)-invasive locoregional recurrence and will have very limited impact on breast cancer mortality. Informed consent is a major therapeutic goal, and better definition of low risk situations remains the main challenge for future research.

\section{Adjuvant Radiotherapy}

Due to new results recently published from prospective randomized clinical studies on radiotherapy, the AGO commission Mamma has reevaluated 2 current questions relevant for clinical practice: i) Can the duration of the adjuvant radiation therapy after breast-conserving therapy be shortened by hypofractionation schemes and do they represent a new standard for postoperative radiotherapy among patients with early breast cancer $[117,175,209,210,243,248]$ ? ii) Is it possible to define subgroups after breast-conserving therapy wherein an only partial breast irradiation (PBI) can be applied optionally using an increased individual dose thereby reducing the duration of the treatment (accelerated partial breast irradiation, APBI)? Is it possible, in an even more extreme form, to perform only a single intraoperative radiotherapy (IORT) which is safe and does not endanger treatment results so far obtained $[133,168,172,183,230]$ ?

With regard to question 1 , the following different concepts of hypofractionation were examined among patients without locoregional involvement of lymph nodes: i) application of higher partial doses per fraction with a correspondingly lower number of fractions and application of lower cumulative doses in a shorter period of treatment (3 vs. 5 weeks) (Canadian trial, START B-trial); ii) application of diverse partial and cumulative doses within identical periods of treatment (START A-trial, RMH/GOG-trial). With the exception of the Canadian study with a median follow-up period of 10 years [243], these studies have - with respect to a possible equivalence of normofractionated as opposed to hypofractionated radiation therapy (cumulative doses of 39-42.9 Gy applied in partial doses of 2.65-3.3 Gy over a time period of 3 or 5 weeks) - only a short median follow-up periods of 5-6 years (START trials). The evaluations as to the hypofractionated radiation therapy have so far yielded: i) no rise of the rate of local relapses; ii) at partial doses lower than $3.3 \mathrm{~Gy}$ and cumulative doses lower than 42.9 Gy no negative effects on cosmesis or reinforcements of the radiogenic toxicity; iii) no increased incidence of other radiogenic long-term effects; iv) 1 in 2 studies showed a survival advantage at a shortened period of treatment. These results from the prospective randomized studies on hypofractionation have internationally led to different assessments and continue to dominate a controversially led discussion. In Canada and Great Britain, hypofractionated radiation therapy is valued as the 'new standard' and assessed as being of equal value to normofractionated radiation therapy [105, 122, 191, 243], and is generally recommended in the updated guidelines. In other national guidelines it is considered an option for certain groups of patients $[15,32,164]$. It should be pointed out, however, that merely the Canadian study is based on long-term results (10 years of follow-up) [243]. The Cochrane Collaboration reassessed the prospective randomized studies on hypofractionation and changed their conclusions in its 'systematic review' of November 2010 [117]; Compared with 2008, Cochrane performed a downgrading to 'studies with low to middle quality' and criticised that with respect to an assessment of equal value compared to the established 'normofractionated' radiation therapy, only a very small number of patients have been treated within randomized studies and observed for more than 5 years. Longer follow-up periods are required before definite conclusions can be drawn. The AGO commission therefore judges a hypofractionated radiation therapy '+/-' for selected patients.

With regard to question 2, from numerous analyses on the location of local relapses it is known that the by far biggest percentage appears in the so-called index quadrant, i.e. in an immediate local neighborhood to the primary tumor [227]. This knowledge led to 2 new radio-oncologic treatment concepts which are applied in the form of accelerated radiation 
therapy (APBI): i) Giving priority to the boost radiation therapy to be applied to the primary tumor area, which shortens the duration of the radio-oncologic therapy: this may be achieved either by a modification during the homogeneous radiation treatment of the complete breast (whole-breast irradiation, WBI) by administering a so-called 'integrated boost radiation therapy' additionally and simultaneously in the form of a percutaneous PBI therapy [111] or by applying intra-operative partial breast radiation treatment (IORT) during surgical tumor excision as 'prior' boost radiation therapy (= 'anticipated' boost) [228]; b) The limitation of the irradiation on the primary tumor area only (definitive PBI) under renunciation of a WBI: different concepts with different radiation qualities and technical methods to PBI and with application of higher partial doses (APBI) have been worked out. These methods correspond to a great deal with those which are used in PBI as boost radiation therapies following WBI $[168,172)$. For selected patients the first results of phase I/II trials seem to be comparable with those of the WBI; there are, however, hardly any data on phase III trials. Results of phase III will not be available before several years. The radiobiological equi-efficacy of the different APBI techniques and the different toxicities of the individual APBI methods are unclear. This also applies to the special case of an only IORT $[229,235]$. In this case, too, the follow-up periods are still too sparse for patients given only APBI treatment within prospective randomized studies; many studies have not been finished yet. The AGO commission points out that WBI still represents the gold standard and that an APBI as a prior ('anticipated') or postoperative boost radiation therapy is suitable for supporting WBI, giving it a grade of recommendation of ' + '. As the only radiation therapy, APBI is still judged as being experimental. Its application as only IORT outside studies is not recommended and therefore rated '-'. Key questions for a successful application of the APBI to be clarified still further concern patient selection, fractionation concepts, scheduled volume definition, optimal fractionation and dosage schemes, imaging and pathology, evidence from clinical studies regarding efficacy, quality of life long-term results, cosmesis, cost/benefit ratio, and longterm toxicity.

\section{Therapy Side Effects}

This chapter was expanded with a special focus on therapyrelated behavioral symptoms like fatigue, sleep disturbances, depression, and cognitive impairment. These behavioral symptoms affect a considerable number of breast cancer patients. They are typically underreported and undertreated, and might adversely affect quality of life [22]. Most often the occurrence of these symptoms is therapy-related. Several studies show that behavioral symptoms improve over time. However, sometimes they might persist for several years.
Several therapeutic interventions have shown to improve these symptoms. For instance, behavioral and psychological interventions [208] as well as physical exercise [152] have demonstrated efficacy in reducing fatigue among breast cancer patients and survivors. Behavioral therapies have demonstrated efficacy in the treatment of insomnia, including insomnia secondary to medical conditions [18]. Of note, they are as effective but longer lasting than pharmacotherapy [204]. Depression can be treated with psychological interventions and pharmacotherapy if indicated [161]. Regular exercise participation was shown to improve depression in breast cancer survivors [38]. Concerning cognitive impairment often referred to as 'chemobrain', cognitive behavioral therapy might lead to significant improvements in self-reported cognitive function, quality of life, and standard neuropsychological test performance [76].

\section{Breast Cancer: Specific Situations}

Adjuvant treatment in young women frequently adversely affects fertility. Before the start of treatment patients need to be informed about the possibility of compromised ovarian function due to adjuvant treatment, and counseling for fertility protection should be offered. In Germany, the FERTIPROTECT Project is a platform to gain advice on how and where to get information [247].

Given the increasing average age of pregnant women, the coincidence of a breast cancer diagnosis and the woman being pregnant or nursing is becoming more frequent. It is thus important to increase the knowledge about breast cancer in this specific situation. In a registry initiated by the GBG 313, women independent from treatment or gestational age were included. So far, 289 of them could be analyzed. Primary objective was fetal outcome 4 weeks after delivery. As expected the tumors were more advanced with roughly half of the patients having a node-positive T2 tumor. More than $50 \%$ of the patients received mostly anthracycline-based chemotherapy during pregnancy. Fetal outcome was comparable between the groups with or without chemotherapy, as was the outcome of patients having chemotherapy during or after delivery. The authors conclude that premature delivery which may increase fetal morbidity does not improve the outcome of pregnancy-associated breast cancer, and patients should be treated as closely as possible to standard recommendations for non-pregnant women [137].

Radiotherapy of the breast reduces the local relapse rate and is usually part of breast-conserving treatment. However the risk of local relapse varies distinctively amongst different patient populations. Recently the long-term results of a randomized phase III trial investigating the role of radiotherapy in elderly patients with breast-conserving surgery was reported. Patients 70 years or older with a clinically negative axilla, T1 tumors, breast-conserving surgery, and hormone receptor- 
positive tumors were randomized to tamoxifen and radiation or to tamoxifen alone. Distant DFS and OS at 10 years were without significant difference between the groups. Local relapse was rare but higher in the non-radiation arm (breast 2 vs. $9 \%$; axilla 0 vs. $3 \%$ ). In a selected low-risk population (T1, N0) of elderly patients ( $<70$ years) with ER-positive disease, radiotherapy may be omitted when endocrine treatment with tamoxifen is planned [116].

\section{Supportive Care (Supportive Therapy)}

The treatment of anemia in the context of cancer experienced an important addition by the introduction of erythropoiesisstimulating factors (ESF). If the use of ESF is considered, adjuvant and palliative setting should be strictly distinguished. In the adjuvant setting a disadvantage in terms of DFS and OS can not be ruled out safely by the use of ESF (LoE 1bA) [74]. Only when using a dose-dense chemotherapy ESF can be used as primary prophylaxis ( $\mathrm{LoE} 1 \mathrm{bA}, \mathrm{AGO}+$ ) [159]. A target level of hemoglobin of 11-12 g/dl should be aimed for $(\mathrm{LoE} 1 \mathrm{aA}, \mathrm{AGO}+)$ and an increased risk of thromboembolic complications due to ESF application has to be considered (LoE 1aA).

Use of colony-stimulating factors should be mandatory if the risk of chemotherapy-induced febrile neutropenia is $>20 \%(\mathrm{LoE} 1 \mathrm{aA}, \mathrm{AGO}++)$ or $10-20 \%$ in the presence of additional risk factors such as age, prior events of febrile neutropenia, or extensive radiotherapy of bone metastases $(\mathrm{LoE} 3 \mathrm{bC}, \mathrm{AGO}+)$ [240].

The use of dexrazoxane for cardioprotection during anthracycline-containing chemotherapy is basically possible, however, a negative interaction with anthracyclinces and a reduction of efficacy can not be excluded ( $\mathrm{LoE} 1 \mathrm{aB}, \mathrm{AGO}+/-$ ). In contrast the use of dexrazoxane for anthracycline extravasation is recommended ( $\mathrm{LoE} 2 \mathrm{bB}, \mathrm{AGO}+$ ) [122, 231]. Antiemetic therapy using neurokinin-1 receptors (LoE 2aB, $\mathrm{AGO}+)$, dexamethasone $(\mathrm{LoE} 1 \mathrm{aA}, \mathrm{AGO}+)$, serotonin antagonists (LoE 2aB, AGO +) and/or metoclopramide (LoE $3 \mathrm{bC}, \mathrm{AGO}+$ ) should be applied subsequent to assessment of the emetogenic potential of a chemotherapy protocol (LoE $5 \mathrm{D}, \mathrm{AGO}++)$. A newly emerged lymphedema of the arm should be considered as a clinical sign of a locoregional recurrence until proven otherwise ( $\mathrm{LoE} 1 \mathrm{aA}, \mathrm{AGO}++)$. After excluding a thrombotic event measures such as physiotherapy (LoE 1aA, AGO ++), compression stockings (LoE 1aA, $\mathrm{AGO}++)$, or electrical stimulation $(\mathrm{LoE} 2 \mathrm{bB}, \mathrm{AGO}+)$ can be fully recommended. In rare cases, laser therapy (LoE 3bC, AGO +/-) or surgery ( $\mathrm{LoE} 4 \mathrm{D}, \mathrm{AGO}+/-$ ) might be an option. Prophylaxis of infection in chemotherapy by using antibiotics as single or combination therapy depends on the expected duration of neutropenia.

Other adverse effects such as mucositis, constipation, and diarrhea (especially with the use of targeted agents) must be detected early and treated vigorously in order to obtain patient compliance.

\section{Breast Cancer Follow-Up}

Modern follow-up care is still marked by only a minimum of 'machine-aided' diagnosis and an emphasis on clinical examination and 'narrative-based medicine'. In addition to early recognition of locoregional recurrences, contralateral breast carcinomas, and other secondary carcinomas, diagnostic procedures tend to be solely symptom-oriented. Other machineaided diagnostic measures, including tumor-marker detection and intensified early recognition, do not currently offer patients any benefit in relation to OS [106, 193]. In addition to the direct costs of such procedures, other costs that need to be taken into account include those for clarifying unclear findings, for increased numbers of complications resulting from interventional diagnostic measures, side effects of treatments in the asymptomatic stage with questionable or unclear benefit, and reduced quality of life due to anxiety related to unclear findings that require check-ups or show unexplained increases in tumor marker levels. The new treatment options available for patients who have breast carcinoma with distant metastases, as well as modern risk classification methods (e.g., gene expression profiling), may in the future change the value of intensified follow-up. However, no changes are currently required with regard to diagnostic methods and follow-up intervals in comparison with last year's recommendations.

Changes or additions to the recommendations apply in particular to the area of interaction between physicians and patients regarding compliance and lifestyle. There are increasingly good data available on the influence of excess weight on DFS and breast cancer-specific mortality rate, particularly in cases of obesity with a BMI $\geq 30 \mathrm{~kg} / \mathrm{m}^{2}$. Increasing weight is directly related to the mortality resulting from breast cancer. It has been shown in retrospective studies that in comparison to patients with a BMI $<25 \mathrm{~kg} / \mathrm{m}^{2}$, those with a BMI $\geq 30 \mathrm{~kg} /$ $\mathrm{m}^{2}$ have a significant $46 \%$ increase in the risk of developing distant metastases within the first 10 years and a $38 \%$ increase in the risk of death due to breast carcinoma after 10 years or more following the primary diagnosis [71]. Continuing physical exercise and keeping weight within the normal range lead to a significant improvement in quality of life, less fatigue, and better physical function. Topics such as physical exercise and weight reduction should therefore be addressed during follow-up appointments with the patients, and contact with support services should be established for them (LoE $2 \mathrm{bB}, \mathrm{AGO}+$ ).

In view of the increasingly complex adjuvant treatments that are being administered, as well as longer treatment periods, it is becoming increasingly important to carry out continuous evaluations of any adjuvant treatment that may still be in progress. This applies in particular to endocrine therapies 
- e.g., with tamoxifen or AI - due to the long treatment period (at least 5 years, or including extended adjuvant therapy up to 10 years), as well as the side effect profile which often leads to patients withdrawing from treatment. Endocrine therapies can only be as good as the use that is made of them. Non-adherence (i.e., taking less than $80 \%$ of the planned dosage) and non-persistence (i.e., premature interruption of an endocrine treatment) lead to a significant reduction in OS (hazard ratio (HR) 1.26 or 1.49 , respectively) due to lack of compliance $[108,110]$. Only $40-60 \%$ of patients complete their endocrine therapy as planned. Predictive factors for withdrawal from endocrine therapy include younger age ( $<50$ years) and also older age ( $\geq 75$ years), breast-conserving therapy (versus ablation), prescription of small pack sizes of medications, larger supplementary charges for medications (particularly in older patients), and the presence of comorbid conditions (2 or more) [109]. During the follow-up consultation, patients should receive detailed information about compliance with endocrine therapies, mentioning the above predictive factors; and possible reasons for withdrawal, such as side effects, should be discussed (LoE 2bB, AGO +).

Compliance is also an essential part of follow-up care itself. The actual usage of the recommended measures often stands in contrast to patients' desire to receive intensified follow-up care. The annual usage of mammography is only in the range of $20-67 \%$ [113]. While $80 \%$ of patients take advantage of mammography during the first year of follow-up, in the 5th year the rate is only $63 \%$. Only $33 \%$ of patients take advantage of mammography in each of the first 5 years. There is therefore an urgent need to provide patients in a targeted way with the information that they need to take advantage of the truly well-established examination measures at the recommended intervals ( $\mathrm{LoE} 2 \mathrm{aB}, \mathrm{AGO}+$ ).

\section{Early Detection and Diagnosis}

Considering screening, early detection, and diagnosis, there were no changes and modifications of the current recommendations and standards compared to 2010.

\section{Breast Cancer Risk and Prevention}

Increasingly important aspects in familial breast cancer affect new risk genes, new therapeutic concepts including PARP inhibitors and platinum-based chemotherapy and prophylactic surgery. After the failure of large linkage studies to identify further high-risk genes, genome-wide association studies provided evidence for the existence of low-penetrance genetic risk variants that may act in concert and are transmitted via a polygenetic trait (e.g., [64]). Although many low-penetrance variants exhibit high minor allele frequencies, relative risks are low and can only explain a small part of the remaining fa- milial aggregation of breast cancer so far. Recently, $R A D 51 C$ was identified as the $3 \mathrm{rd}$ high-risk gene for breast and ovarian cancer that is however rarely mutated [153]. Therefore $R A D 51 C$ constitutes a proof of concept for the existence of many more yet very rarely affected high-risk genes. These genes may be identified by large-scale exomic sequencing efforts.

PARP inhibitors impair base excision repair that leads to persisting single strand breaks (SSBs) that turn into double strand breaks (DSBs) in the replication fork during mitosis $[26,73]$. In the case of a $B R C A$-deficient tumor, these DSBs can not be repaired leading to cell apoptosis (concept of synthetic lethality). Phase I and II studies with PARP inhibitors in the metastatic stage provide evidence that indeed the genetically defined subgroup may benefit from PARP inhibitor treatment $[9,221]$. Similarly chemotherapeutic agents that form DNA-interstrand crosslinks thereby inducing DNA DSBs such as platinum derivates seem to confer high chemotherapeutic sensitivity in $B R C A$-deficient tumor cells [186, 215]. However, clinical data are still limited and restricted to case presentations, retrospective studies, and very small prospective studies [30, 189]. Therefore, the AGO study group strongly recommends further clinical studies in the genetically defined subgroup of patients like the UK-based BRCA trial (PI Andrew Tutt Royal Marsden, Eudrac-No 2004-001496-20).

Recently, there has been a steep increase in the demand for prophylactic mastectomy not only in healthy $B R C A 1 / 2$ mutation carriers but also in $B R C A 1 / 2$ mutation carriers already affected by unilateral breast cancer that harbor a strongly increased risk for contralateral breast cancer and in women with sporadic breast cancer who obviously overestimate their risks $[125,166]$. Several studies provided data that prophylactic oophorectomy, antihormonal treatment, affected gene, modifier genes, and age of onset of first breast cancer all may influence the risk of first or second breast cancer [6, $64,59,97,154]$. Therefore, comprehensive and non-directive risk counseling is a prerequisite prior to the decision for or against prophylactic mastectomy. Also, further studies in large collectives are needed to specify first and contralateral breast cancer risk in women tested negative for $B R C A 1 / 2$ mutations.

\section{Locoregional Recurrence}

On average $10 \%(2-20 \%)$ of patients who undergo breastconservation surgery and radiation therapy will subsequently develop ipsilateral breast tumor recurrence. Isolated recurrences in the ipsilateral supraclavicular fossa fare as well as isolated chest wall recurrences, whereas locoregional recurrences of any site fare worse if the supraclavicular fossa is additionally affected: 3 -year OS has been determined as only $49 \%$ [188]. 
To avoid 'overtreatment' or 'undertreatment' and to prevent complications, the AGO recommends restaging in all patients with resectable recurrences. Routine positron emission tomography (PET) is not recommended [216].

Performing SLNB during relapse surgery (second SLNB) in a $\mathrm{cN} 0$ situation after previous SLNB is technically feasible [56]. A repeat breast-conserving therapy demands tumor-free margins and subsequent repeat radiotherapy, e.g. interstitial brachytherapy. However, the indication for second lumpectomy should be reserved for suitable patients. Repeat irradiation of the chest wall and breast for recurrent breast cancer is feasible. Addition of hyperthermia (special expertise required) improves clinical response rate, particularly in preirradiated superficial areas [234].

\section{Prognostic and Predictive Factors}

Circulating tumor cells in the peripheral blood have found to be of prognostic relevance in primary breast cancer patients [187] and to be predictive of metastatic disease spread [89]. Centrally reviewed Ki-67 labeling index is of prognostic and predictive value for postmenopausal women with endocrine responsive breast cancer [237]. Immune response in the form of tumor-associated lymphocytes is an independent predictor of clinical response to neoadjuvant chemotherapy [54].

\section{Endocrine Therapy in Metastatic Breast Cancer}

Hormonal therapy remains the treatment of first choice for metastatic patients with endocrine responsive breast cancer. In this context the sequential use of the available drugs depends on the adjuvant pretreatment. Fulvestrant plays an increasing role in the treatment of $\mathrm{MBC}$ according to the implementation of $\mathrm{AI}$ into the adjuvant setting. In addition to the approved dose of $250 \mathrm{mg}$ by intramuscular injection, other dose regimens have also been investigated as some studies suggested that the $250 \mathrm{mg}$ dose may not be optimal. A highdose (HD) regimen of fulvestrant (500 mg once a month plus $500 \mathrm{mg}$ on day 14 of month 1) was compared with anastrozole in a phase II trial as first-line treatment of hormone receptorpositive MBC [190]. The primary endpoint, clinical benefit rate, was not statistically significantly different between treatment arms (72.5\% with fulvestrant HD and $67.0 \%$ with anastrozole). However, fulvestrant HD was associated with significantly longer time to progression (TTP) versus anastrozole (median TTP not reached vs. 12.5 months; HR 0.63; 95\% confidence interval (CI) 0.39-1.00; $\mathrm{p}=0.0496$ ).

As mentioned earlier, a LD regimen has also been evaluated. The EFECT trial showed that this regimen of fulvestrant was active after failure of a non-steroidal AI. Two recent trials assessed the efficacy of different fulvestrant dosing regimens. The CONFIRM trial compared the approved fulvestrant dose (fulvestrant AD) with the HD regimen in 736 patients with hormone receptor-positive MBC who had progressed on prior endocrine therapy. Fulvestrant HD was associated with significantly longer progression-free survival (PFS) than the fulvestrant AD regimen (HR 0.80; 95\% CI $0.68-0.94 ; p=0.006)$. Median PFS was 6.5 months with the HD regimen and 5.5 months with fulvestrant AD. Objective response and clinical benefit rates were similar with both doses [57]. As a result, fulvestrant HD is now the approved dose for use in Europe. The FINDER II trial compared fulvestrant $A D$, fulvestrant $H D$ and fulvestrant $L D$ regimens in 144 postmenopausal women with advanced breast cancer after 1 prior endocrine therapy [185].

Comparison of data across the 3 treatment arms showed that fulvestrant AD, HD and LD had statistically similar efficacy, although in all parameters AD had numerically lower results. Objective response rate in the AD, LD and HD arms was $8.5,5.9$ and $15.2 \%$, respectively. Median TTP was numerically longer for the HD and LD arms (6.0 and 6.1 months, respectively) versus AD (3.1 months). Since this was a small trial with 3 arms, it is difficult to draw definite conclusions.

As such, fulvestrant is a suitable treatment option for some patients following failure of treatment with prior endocrine therapy, even if questions remain regarding the optimal dosing regimen between LD and HD. Additionally, based on currently available data, HD fulvestrant appears to be as well tolerated as LD fulvestrant.

\section{Cytotoxic Therapy in Metastatic Breast Cancer}

For changes, see www.ago-online.de.

\section{Specific Sites of Metastasis}

For changes, see www.ago-online.de.

\section{Bone Metastases}

There were no clinically relevant changes compared to 2010.

\section{Central Nervous System Metastases in Breast Cancer}

Despite major improvements in the therapeutic options available for patients with advanced breast cancer, brain metastases remain a challenging clinical problem. As systemic therapies for breast cancer have improved and OS lengthens, it appears that the incidence of brain metastases is increasing, a greater proportion of patients have controlled extracranial disease at the time of their central nervous system (CNS) diagnosis, and CNS recurrence after initial treatment is 
becoming more frequent. In contrast to the clinical estimates, autopsy series indicate that up to $40 \%$ of patients have CNS involvement at the time of death. Biological risk factors for brain metastases include women under the age of 50 years, ER-negativity, high-grade tumors, overexpression of HER1 and HER2, basal like tumors, high proliferation index Ki-67 $(>15 \%)$, successful trastuzumab therapy for extracranial lesions, and successful adjuvant chemotherapy. Brain metastases mostly appear metachronously often as single metastasis. Symptoms are nausea, emesis, headache, and focal neurologic dysfunction. In patients presenting with suspicious neurological signs evaluation with computed tomography or contrast-enhanced magnetic resonance imaging (MRI) is indicated. In cases of meningeal carcinomatosis, lumbar puncture will detect tumor cells in $70 \%$. Generally, median survival of patients with brain metastases is very poor and in the range of several months. Predictive factors for prolonged survival include Karnofsky performance status greater than 70 , solitary brain metastases, systemic tumor control, longer DFS and ER positivity. Treatment options for patients with solitary brain metastases are whole-brain radiotherapy (WBRT) plus stereotactic radiosurgery (SRS) with improvement of local control and survival time ( $\mathrm{LoE} 2 \mathrm{aB}, \mathrm{AGO}++)$, WBRT $(\mathrm{LoE} 2 \mathrm{bB}, \mathrm{AGO}+$ ), SRS (lesions $<3 \mathrm{~cm})+/-$ WBRT $(\mathrm{LoE} 2 \mathrm{bB}, \mathrm{AGO}++)$ and SFRT (LoE $3 \mathrm{bB}, \mathrm{AGO}+/-)$. Standard treatment for patients with multiple brain metastases is WBRT (prolonged radiotherapy: LoE 3bB, AGO ++; $10 \times 3$ Gy: LoE 2bB, AGO +) combined with corticosteroids against symptomatic perifocal edema (LoE 1aA, AGO ++). Radiotherapy is a treatment option in selected cases (LoE 3bC, AGO +/-). Chemotherapy alone is evaluated only in phase II studies ( $\mathrm{LoE} 3 \mathrm{aD}, \mathrm{AGO}-$ ). Corticosteroids alone are not the treatment option of choice ( $\mathrm{LoE} 3 \mathrm{aB}, \mathrm{AGO}-$ ). In the situation of radioresistance, both chemotherapy (LoE $3 \mathrm{aD}$, AGO +/-) and lapatinib +/- capecitabine (HER2-positive breast cancer) (LoE 2bB, AGO +/-) are options of individual therapy. Options of local treatment of leptomeningeal metastases are intrathecal or intraventricular therapy with methotrexate 10-15 mg 2-3 times/week (LoE 2bB, AGO ++), liposomal cytarabine (50 mg twice a week) ( $\mathrm{LoE} 3 \mathrm{bC}, \mathrm{AGO}++)$, thiothepa (LoE 3bC, AGO +), corticosteroids (LoE 4D, $\mathrm{AGO}+/-)$, or focal radiotherapy in the case of lesions greater than $7 \mathrm{~cm}(\mathrm{LoE} 4 \mathrm{D}, \mathrm{AGO}+)$, alternatively WBRT (LoE 4D, $\mathrm{AGO}+)$.

\section{Targeted Agents}

Since endocrine therapy as one of the most established options of targeted therapy in the systemic treatment of breast cancer has been introduced decades ago, the identification of new drugs and components had tremendous impact on the treatment options in metastatic disease. Newer treatments have improved survival for patients with $\mathrm{MBC}$ over the last
2 decades, and a battery of new cytotoxic and targeted therapies is continuing to enhance this trend. Recent data and ongoing research in this area, and also new classes of targeted therapy that are undergoing clinical evaluation, are detailed in this chapter.

\section{Targeting the HER2 Family}

At present, the majority of treatment options is available for blockade of the HER2 receptor either at the extracellular or the intracellular domain, or using both options. HER2 is amplified and/or overexpressed in up to $20 \%$ of human breast tumors and is associated with a poorer prognosis [42, 201]. A new generation of HER2-targeting agents has been developed, and alternative cytotoxic combination strategies are currently being explored. One such HER2-targeting agent, trastuzumab, has demonstrated synergistic activity against breast cancer cells overexpressing HER2 when used in combination with chemotherapy. Besides selecting the right treatment, proper testing of the target remains the key issue [245].

\section{The Newer Generation of HER2-Targeting \\ Monoclonal Antibodies}

\section{Trastuzumab-MCC-DM1}

The additive or synergistic potential of trastuzumab, in combination with microtubule targeting agents, has resulted in trastuzumab-MCC-DM1 (T-DM1) - a novel antibody/drug conjugate that uses trastuzumab to specifically deliver the cytotoxic maytansinoid antimicrotubule to HER2 cells. Mechanistically, DM1 binds to microtubules in a manner similar to that of vinca alkaloids [128]. The first-in-human phase I, multicenter, open-label, dose-escalation study of single-agent T-DM1 in patients with HER2-overexpressing MBC (who had previously received a trastuzumab-containing chemotherapy regimen) demonstrated that, at the maximum-tolerated dose (MTD) of $3.6 \mathrm{mg} / \mathrm{kg}$ every 3 weeks, T-DM1 was safe and had considerable clinical activity. The clinical benefit rate (CBR: overall response rate (ORR) plus stable disease (SD) at 6 months) among 15 patients treated at the MTD was $73 \%$, including 5 objective responses. Furthermore, the confirmed response rate in patients with measurable disease at the MTD $(n=9)$ was $44 \%$ [128]. Phase II studies of T-DM1 in patients with HER2 MBC who progressed while receiving HER2directed therapy, or who were previously treated with an anthracycline, a taxane, capecitabine, lapatinib and trastuzumab, have demonstrated robust activity, with ORRs in the range of 23.9-39.5\%, as determined by an independent review facility. An open-label, phase III trial (EMILIA) will compare the safety and efficacy of T-DM1 with that of capecitabine in combination with lapatinib in patients with HER2 MBC previously treated with a trastuzumab-based therapy [45]. Currently, T-DM1 is in clinical development for the treatment of 
MBC as both a monotherapy and in combination with other agents such as docetaxel or pertuzumab plus paclitaxel.

\section{Pertuzumab}

Pertuzumab is a novel recombinant humanized monoclonal antibody directed against the highly conserved dimerization domain of HER2, and as such, it inhibits HER2 homo- and heterodimerization. Pertuzumab-mediated blockage of HER2 dimerization inhibits HER family downstream signaling (i.e., the Akt cell survival pathway and the mitogen-activated protein kinase pathway). Preclinical models have demonstrated that, unlike trastuzumab, pertuzumab is able to inhibit tumor growth of cancer cells expressing both low/normal and high levels of HER2. However, in a phase II randomized trial investigating the efficacy and safety of pertuzumab in patients with HER2-positive MBC, the only measurable therapeutic benefit that Gianni et al. [86] observed was SD of a relatively short duration. These data suggest that patients with HER2-positive MBC would be more likely to experience a clinically meaningful response from treatment with pertuzumab [86]. The idea that the combination of pertuzumab and T-DM1 might be a clinically meaningful therapy in MBC came from the single-arm, phase II trial of trastuzumab plus pertuzumab, which demonstrated that the combination was well tolerated and active in patients with HER2-positive MBC, who had progressed during trastuzumab therapy [13]. Ongoing clinical trials continue to investigate pertuzumab for the treatment of $\mathrm{MBC}$ in combination with trastuzumab, T-DM1, docetaxel, and paclitaxel. Because preclinical evidence suggests resistance may, in many cases, arise from aberrant signaling downstream of HER2 (e.g., constitutive phosphoinositide 3-kinase (PI3K) signaling), it is not yet clear whether pertuzumab will be effective in trastuzumab-resistant tumors [163].

\section{Small-Molecule Inhibitors of the HER2 and EGFR Pathways}

\section{Lapatinib}

Lapatinib inhibits intracellular receptor phosphorylation of HER2 and the epidermal growth factor receptor (EGFR) [31, 126], whereas trastuzumab targets the extracellular domains of HER2. These distinct mechanisms of action may account for the lack of cross-resistance between trastuzumab and lapatinib and for their in vitro synergy [126]. In a phase III trial of lapatinib in combination with trastuzumab compared with lapatinib alone in 296 heavily pretreated patients with HER2 MBC, who had experienced progression on a median of 3 prior trastuzumab-based therapies (the EGF104900 trial), the PFS interval was significantly longer with the combination than with lapatinib alone (median 3.0 vs. 2.0. months; HR 0.77 ; 95\% CI $0.6-1.0 ; \mathrm{p}=0.029)$. However, there was no significant difference in the ORR $(10.3$ vs. $6.9 \%$; $=0.46)$, and although there was a trend toward a longer OS duration for patients in the combination arm, this difference was also not significant (12.9 vs. 9.8 months; HR 0.75; 95\% CI 0.5-1.1; $\mathrm{p}=0.106)[21]$.

\section{Neratinib}

Neratinib, which irreversibly inhibits the kinase activity of HER2 and EGFR, showed a $32 \%$ response rate as a single agent in trastuzumab-pretreated MBC patients [219]. As such, this agent is currently being studied in combination with capecitabine, paclitaxel, or vinorelbine in phase I/II trials of patients with HER2 MBC. Moreover, a large randomized phase II trial designed to evaluate the safety and efficacy of neratinib in advanced HER2 breast cancer is ongoing (// clinicaltrials.gov, identifier NCT00300781). The estimated enrolment is 136 patients, and the primary endpoint is the 16week PFS rate.

\section{Antiangiogenic Therapy}

\section{Bevacizumab}

Bevacizumab is a humanized monoclonal antibody that inhibits the vascular endothelial growth factor (VEGF), depriving tumors of a vascular supply and inhibiting endothelial proliferation [157]. In a prospective, randomized, but not blinded phase III trial in the first-line treatment of MBC patients, the combination of bevacizumab and paclitaxel led to a significantly longer PFS interval than with paclitaxel alone (median 11.8 vs. 5.9 months; HR 0.60; p < 0.001) and higher ORR (36.9 vs. $21.2 \%$; p 0.001 ) with minimal toxicity, not unlike safety profiles reported in previous randomized trials [157]. However, there was no significant difference in OS between the groups (median 26.7 vs. 25.2 months; HR 0.88; $\mathrm{p}=0.16$ ). Additional confirmatory trials combining this agent with docetaxel (AVADO: Avastin and Docetaxel) [156] or with anthracyclines and taxanes or capecitabine (RIBBON-1: Regimens in Bevacizumab for Breast Oncology) have demonstrated enhanced efficacy in the first-line setting [169]. However, addition of bevacizumab to chemotherapy did not result in longer OS in either the AVADO or RIBBON-1 trial. A meta-analysis of OS from the phase III randomized trial evaluating bevacizumab in combination with paclitaxel (E2100) and the AVADO and RIBBON-1 trials (a total of 2,447 patients) confirmed the significant PFS benefit for bevacizumab in combination with chemotherapy, compared with chemotherapy alone, for the first-line treatment of MBC patients (median 9.2 vs. 6.7 months; HR 0.64; $\mathrm{p}<0.0001$ ) [169]. Although OS was not significantly different between treatment arms (median 26.7 vs. 26.4 months; HR 0.97; 95\% CI 0.86-1.08; $\mathrm{p}=0.56$ ), the 1 -year survival rate was greater for bevacizumab in combination with chemotherapy than for chemotherapy alone (81.6 vs. $76.5 \%$; $\mathrm{p}<0.003$ ), suggesting an early benefit at 1 year. Given the marginal benefit and toxicity of bevacizumab, the VEGF target will need to be defined specifically for each particular 
subtype of breast cancer to maximize benefits in treated patients.

\section{Poly(ADP-Ribose) Polymerase Inhibitors}

The clinical efficacy of poly(ADP-ribose) polymerase (PARP) inhibitors is attributed to their ability to take advantage of the impaired mechanism of DSB repair used by some tumors. DNA lesions such as SSBs and DSBs are common byproducts of normal cellular metabolism, and may also result from exposure to harmful environmental agents. In brief, 4 DNA repair mechanisms are responsible for repairing these lesions: i) base-excision repair (BER), ii) nucleotide-excision repair (NER), iii) mismatch repair (MMR), and iv) recombinational repair (with homologous recombination and non-homologous end joining (NHEJ)) [222]. When SSBs occur, they are repaired using the intact complementary strand as a template by BER, NER, and MMR. A key component of the BER pathway, PARP1 is the most important member of the PARP family of enzymes. DSBs are primarily repaired by homologous recombination - almost error free - and NHEJ which is more susceptible to error. The tumor suppressor proteins BRCA1 and BRCA2 play a pivotal role in the repair of DSBs by homologous recombination. However, in BRCA-deficient cells that have accumulated DSBs, loss of BRCA function renders the cell incapable of repairing DSBs by homologous recombination. Instead, the cell defaults to the error-prone NHEJ pathway, resulting in genomic instability that ultimately leads to cell death. Thus, patients whose tumors are BRCA-deficient are ideal candidates for treatment with PARP inhibitors in the clinical setting. Because triple-negative breast cancer shares pathologic and gene expression profiles with BRCA-associated breast cancers, both subtypes are currently being evaluated in clinical trials of PARP inhibitors - as single agents and in combination with chemotherapy. In a phase II trial of heavily pretreated patients with recurrent, measurable, chemotherapy-refractory $B R C A$ mutation associated breast cancer, the PARP inhibitor olaparib (400 mg twice daily and subsequently $100 \mathrm{mg}$ twice daily) demonstrated measurable single-agent efficacy, with an ORR as high as $41 \%$ [220].

Like BRCA-associated breast cancers, triple-negative breast cancer is sensitive to PARP inhibition in the presence of platinum-based chemotherapy. In the first randomized phase II trial of a PARP inhibitor in triple-negative MBC patients, iniparib (5.6 mg/kg intravenously biweekly) on days 1 , 4,8 , and 11 every 3 weeks was combined with chemotherapy (gemcitabine $1,000 \mathrm{mg} / \mathrm{m}^{2}$, and carboplatin area under the curve (AUC) 2, both given on days 1 and 8 every 3 weeks) to determine the effect on PFS and OS. The addition of iniparib to gemcitabine plus carboplatin not only resulted in a longer PFS duration (median 7.0 vs. 2.9 months; HR 0.30; 95\% CI $0.15-0.59 ; \mathrm{p}<0.0003$ ) but also resulted in a longer OS time (median 8.5 vs. 5.6 months; HR 0.24; 95\% CI 0.09-0.61; $\mathrm{p}<0.0012)$ and higher CBR (52 vs. $12 \%$; p < 0.0012) than with gemcitabine plus carboplatin alone [170]. Updated results presented at the 2010 ESMO Congress showed that, with extended follow-up, the combination led to significantly longer OS than with gemcitabine plus carboplatin alone (12.2 vs. 7.7 months; HR 0.5; $95 \%$ CI $0.30-0.82 ; \mathrm{p}<0.005)$. A phase III, randomized, multicenter trial of gemcitabine plus carboplatin with or without iniparib in patients with triple-negative breast cancer is now complete and awaits assessment (//clinicaltrials.gov; identifier NCT00938652). Importantly, on January 28, 2011, a press release from Sanofi-Aventis and its subsidiary BiPar Sciences stated that this phase III trial did not meet the prespecified criteria for significance for the coprimary endpoints of OS and PFS; this has been presented correspondently at ASCO 2011 [171]. However, data from the prespecified analysis in patients treated in the second- and third-line setting do support the findings reported in the phase II trial - longer OS and PFS times. With data to support the efficacy of PARP inhibitors in the clinical setting, researchers have begun to focus on tailoring treatment regimens according to specific triple-negative subtypes.

A translational phase II trial of olaparib in patients with ovarian cancer (BRCA1 and BRCA2 carriers) and triplenegative breast cancer (BRCA1 and $B R C A 2$ non-carriers) demonstrated that, as a single agent, olaparib provided clinical benefit only to patients with ovarian cancer [82]. In that study, the ORR was approximately $41.2 \%$ in 64 women with ovarian cancer, whereas the ORR was zero in 24 patients with triple-negative $\mathrm{MBC}$. These data suggest that triplenegative breast cancer $B R C A 1$ and $B R C A 2$ carriers may be better candidates for therapy with single-agent PARP inhibitors than triple-negative breast cancer $B R C A 1$ and $B R C A 2$ non-carriers.

Because BRCA1-associated triple-negative tumors are more likely to have a deficiency in DNA repair than sporadic triple-negative tumors, Rodriguez et al. [192] designed a study to define a gene expression signature that would facilitate their identification. Such an assay could enable the identification of patients with triple-negative breast cancer, who would have a greater response to neoadjuvant DNA-damaging agents than patients with a different expression profile.

In summary, over the last decade the most impressive advantage has been achieved in HER2/neu-overexpressing MBC. Introduction of bevacizumab as a VEGF-directed targeted treatment remains an issue on debate. Some of the novel therapeutics to the breast cancer armamentarium resulted in prolongation of survival for patients with MBC. At present, data have been released that in some cases the early onset of a dual targeting approach might be very promising, such as trastuzumab and pertuzumab or trastuzumab and lapatinib. Hopefully, in the coming years, the number of treatment options for MBC patients will continue to increase. Clinical development of new targeted agents of the second 
generation such as neratinib or afitinib might act as irreversible inhibitors or as partners with chemotherapy in specific indications. Specifically, cross-resistance remains a concern because targeted and cytotoxic agents may have different but overlapping mechanisms of action. The safety profiles of cytotoxic and biologic compounds are expected to overlap only in limited ways. It is worth noting, however, that the target proteins of biologic agents that are dysregulated in cancer are commonly essential components of normal cell growth and development. Thus, it is challenging to completely anticipate any potential toxicities associated with inhibiting endogenous levels of these proteins and how this inhibition might exacerbate toxicities associated with chemotherapy (i.e., bevacizumab plus cytotoxic chemotherapy). A better understanding of the molecular interactions between targeted agents and cytotoxic chemotherapy, combined with increasing knowledge of the molecular mechanisms underlying drug resistance, has enabled the rational design of effective combination regimens for the treatment of MBC patients. With these advancements in breast cancer biology, the natural history of MBC now resembles that of a chronic disease for which longer survival and preservation of quality of life are the goals of treatment. The expanding number of possibilities for regimens that combine new anticancer agents may be expected to translate into better outcomes for patients with MBC.

\section{Complementary Therapy in the Context of Integrative Oncology and Hormonal Treatment and Alternatives in Breast Cancer Survivors and Survivorship}

Immediately following diagnosis - at the latest after being discharged from hospital - most cancer patients begin to think about therapies or measures they can undertake in the expectation that the healing process will be supported. These patients require comprehensive guidance as to the possibilities, effectiveness, and side effects of such therapeutic approaches. This requires that physicians responsible for treatment adopt an informed orientation with respect to the expectations of their patients. Not infrequently, interested parties in the patients' social environment exaggerate these expectations unrealistically.

Integrative oncology takes both complementary and conventional oncology into account. Among the positive recommendations in complementary therapy there are, in particular, acupuncture (for postoperative and chemotherapy-induced nausea and vomiting or for the relief of postmenopausal symptoms) [48, 72, 77, 81, 130, 212], early postoperative introduction of exercise for short-term recovery of shoulder flexion [151], and the methods of mind-body medicine [160] with mindfulness-based stress reduction (MBSR) and yoga. MBSR provides patients with strategies for coping with the stresses of daily life and offers them encouragement for self help [150]. MBSR and yoga can improve the quality of life with respect to mindfulness and a reduction of stress, anxiety, and depression [174, 198, 202, 203, 226, 244]. Furthermore, MBSR can help to reduce menopausal symptoms after breast cancer [33, $67,75,176,217]$.

There are many substances which, due to possible interactions, should not be taken during the course of standard oncologic therapy. This includes, for example, large doses of vitamin $\mathrm{C}$ and antioxidants in general [98, 107, 162, 173], proteolytic enzymes [182], thymus and spleen peptides [145, 155], coenzyme Q10 [135, 136, 143], selenium [55, 98], vitamin E, and especially the use of ginseng in cases of hormone receptor-positive breast cancer due to estrogen-like effects $[4,11$, $50,132,206]$.

Various medications can be employed for the relief of postmenopausal symptoms following breast cancer [176]. These are, in particular, selective serotonin reuptake inhibitors (SSRI) and serotonin noradrenaline reuptake inhibitors (SNRI) for reducing hot flashes. The medication of first choice here is venlafaxine [27, 70, 138-141] followed by desvenlafaxine [7, 53, 207], sertraline, and escitalopram due to the minimal interaction via Cyp2D6, for example, with tamoxifen $[8,20,199,200]$. It is important that the dosage of these medications is gradually reduced to prevent a discontinuation syndrome $[58,100]$. Paroxetine, fluoxetine, duloxetine, and bupropion should not be administered as they have a relevant inhibiting effect on tamoxifen $[5,8,123,199]$.

\section{Bisphosphonates and RANKL Antibody Denosumab}

Bisphophonates (BP) are an integral part in the treatment of breast cancer patients with bone metastases. They reduce the prevalence of skeletal-related events (SRE) including fractures, skeletal instability, and loss of skeletal integrity requiring surgery or radiation, spinal cord compression, and hypercalcemia $(\mathrm{AGO}++)$. BP should be continued beyond disease progression $(\mathrm{AGO}++)$ [179].

In the adjuvant setting, BP can be given to prevent tumor therapy-induced bone loss in pre- and postmenopausal women $(\mathrm{AGO}+)$ [66, 108]. In addition, BP improve DFS in postmenopausal breast cancer patients regardless of the type of adjuvant treatment $(\mathrm{AGO}+)$. In premenopausal patients a benefit for DFS could only be demonstrated in those who have received tamoxifen or AI in combination with a GnRHa $[46,66,90]$. Therefore there is currently no rationale for adjuvant $\mathrm{BP}$ in hormone receptor-negative premenopausal patients $(\mathrm{AGO}+/-)$ or hormone receptor-positive patients with cytotoxic and subsequent endocrine treatment $(\mathrm{AGO}+/-)$.

Denosumab is a monoclonal antibody to the RANK ligand. Denosumab (120 mg subcutaneously every 4 weeks) has demonstrated efficacy in reducing the morbidity of bone metastases and prolonging the skeletal event-free survival $(\mathrm{AGO}++)$ [211]. Similiar to the BP, denosumab should be continued beyond disease progression $(\mathrm{AGO}++)$. Deno- 
sumab has recently been approved for use in patients with bone metastases. Denosumab (60 mg s.c. every six months) has already been proven to be effective in preventing and treating therapy-induced bone loss $(\mathrm{AGO}+)$ [68]. Therapyinduced bone loss is a serious concern in pre- and postmenopausal patients with adjuvant treatment for breast cancer (regardless of whether cytotoxic or endocrine treatment or both are applied) [101]. Management of patients should therefore be in compliance with the guidelines of the DVO (Dachverband Osteologie e.V.) Guideline 2009 [62].

Epidemiologic data suggest that breast cancer incidence is reduced in postmenopausal women using oral BP for osteoporosis treatment $(\mathrm{AGO}+/-)$ [39].

\section{Options for Primary Prevention, Modifiable Risk Factors}

Beside hereditary and genetic origins of breast cancer (chapter 'Breast Cancer Risk and Prevention'), the AGO Breast Committee felt that also non-genetic factors play an increasing role in development of breast cancer, and in order to inform about this issue, an additional chapter considering this was attached. We distinguish between modifiable, socially defined, and clearly non-modifiable risk factors [158]. Modifiable risk factors are less breast feeding, high BMI/obesity, steroid hormone therapy, recent oral contraceptive use, hormone therapy in postmenopausal women, alcohol intake, less physical activity. Socially defined risk factors are older age at first full term delivery and lower number of parity. Non-modifiable risk factors are older age, genetic risk factors, family cancer history, personal history of breast lesions (non-proliferative lesions, proliferative lesions with or without atypia, high risk lesions $(\mathrm{ADH}$, lobular intraepithelial neoplasia (LIN)), breast cancer history (DCIS, invasive breast cancer), breast density, chest irradiation, life time number of menstrual cycles (early menarche, late menopause).

Thus, at least modifiable risk factors should be identified and women should be counseled in order to enhance awareness about their behavior with regard to their own health. It seems also to be important to define factors that according to the available evidence do not have influence on breast cancer risk such as diets, vitamins (including phytoestrogens, green tea, etc.), antiperspirants, bras, miscarriages or induced abortion, breast implants, environmental chemicals, tobacco smoke, night work or shift work [65, 83, 246].

With regard to the influence of reproductive life on breast cancer risk, early age at first full term pregnancy (LoE 2bB), higher number of children (LoE 2bB), and long cumulative time of breast feeding ( $>1.5-2$ years; LoE $3 \mathrm{bB}$ ) might be protective. However, this effect seems to be confined to ER/PRpositive disease [47, 142, 144, 149].
Of increasing importance is the Western world type lifestyle that obviously increases the risk of breast cancer. By modifying these factors, breast cancer risk can be reduced substantially. In detail, maintaining normal weight (aimed at BMI $18.5-25 \mathrm{~kg} / \mathrm{m}^{2}$; LoE $2 \mathrm{aB}, \mathrm{AGO}++$ ) is effective in premenopausal (LoE $3 \mathrm{aB}, \mathrm{AGO}++$ ) and particularly in postmenopausal women $(\mathrm{LoE} 2 \mathrm{aB}, \mathrm{AGO}++)$. There are some hints that dietary patterns may play a role and that a Mediterranean, prudent, or healthy diet should be preferred over a Western unhealthy diet ( $\mathrm{LoE} 2 \mathrm{bB}, \mathrm{AGO}+)$. There is accumulating evidence that particularly reduced fat diet reduces the breast cancer risk ( $\mathrm{LoE} 2 \mathrm{aB}, \mathrm{AGO}+$ ), however, less confirmed is the role of vitamins, minerals, trace elements, vegetables, and fruits (LoE 2aB, AGO +/-) [24, 49, 93, 184, 218].

Often underestimated is the role of alcohol consumption. Reduction of alcohol intake reduces the risk of breast cancer substantially and women should be counseled (LoE 2bB, $\mathrm{AGO}++)$. The effect is pronounced for the development of $\mathrm{ER} / \mathrm{PR}$-positive tumors and invasive lobular tumors (LoE 2bB) [83, 124, 134, 214].

It is generally accepted that physical exercise reduces the risk of breast cancer, although current data does not provide homogenous evidence ( $\mathrm{LoE} 2 \mathrm{a}(-) \mathrm{B}$; AGO ++). Physical exercise is defined as the metabolic equivalent to $3-5 \mathrm{~h}$ moderate pace walking per week $[51,79,83]$.

A major issue is the use of hormonal therapy (HRT) for postmenopausal symptoms. Available data suggests that the use of HRT should be avoided ( $\mathrm{LoE} 2 \mathrm{aB}, \mathrm{AGO}++$ ). In detail, particularly avoiding estrogen/progestin combinations reduces the risk of breast cancer substantially (LoE $1 \mathrm{bA}$, $\mathrm{AGO}+$ ). However, enhanced breast cancer risk has not been demonstrated for the use of estrogens only ( $\mathrm{LoE} 1 \mathrm{bA}, \mathrm{AGO}$ +/-) [10, 16, 17, 40, 41, 131].

Finally, an important discussion focuses on the effects of oral contraception. Overall, oral contraception seems not to increase breast cancer risk ( $\mathrm{LoE} 1 \mathrm{aA})$. However, breast cancer risk is slightly increased among current oral contraceptive users (LoE $1 \mathrm{a}(-))$; the effect disappears 5-10 years after stopping oral contraception and is not dependent on the type or composition of the oral contraceptive ( $\mathrm{LoE} 1 \mathrm{a})$.

Of particular interest is that the risk of developing breast cancer may be increased among oral contraceptive users (especially in long term users) if started at a young age before the first full term pregnancy, although the absolute risk and absolute number seem to be still very low for having a generally low incidence at this age $(\operatorname{LoE} 1 \mathrm{a}(-))$.

The risk of breast cancer for users of oral contraceptives is not increased with a family history of breast cancer (LoE 1a). It is possibly slightly increased for $B R C A 1 / 2$ mutation carriers, but current data suggest that this risk is well balanced by reduction of ovarian cancer risk ( $\operatorname{LoE} 1 \mathrm{a}(-))[23,35,43,60$, $61,80,103,104,121,129,147,194,197,236]$. 


\section{Guidebook for Patients 2011}

The AGO guidelines are accompanied by a guidebook for patients (Patientenratgeber), which is also updated annually. It translates the guidelines into a language appropriate for nonprofessionals and is intended to support physician-patient communication and shared decision-making. The changes in the 2011 edition compared to the previous version mirror the statements outlined above. It can be downloaded at $w w w . a g o-$ online.de/index.php? lang=en\&site=mamma_guide_topical .

\section{Disclosure Statement}

The members of the editing committee of these guidelines are specialists in diagnosis, treatment, and research in breast cancer. Most of the members therefore cooperate with the industry. Thus, potential conflict of interest cannot be excluded. In order to minimize potential bias within the statements we followed these pre-defined rules: These guidelines are strictly based on available evidence from the scientific literature; the chapters of each edition were prepared by annually alternating teams of authors; each statement and the correspondent AGO recommendations were thoroughly discussed within the entire group and accepted by majority decisions; each member of the editing committee is required to submit a written declaration of his/her conflicts of interest to an elected internal Conflicts of Interest (COI) committee on an annual basis; members who do not submit a COI declaration may not participate in the guideline preparation. All members of the AGO Breast Committee have submitted their COI report for the past year. Members of the AGO Breast Committee indicated that they have received support (e.g. research funding, lecture or consulting honoraria etc.) from the following entities: Abraxis BioScience Ltd., Amgen GmbH, AstraZeneca GmbH, Bayer-Schering Pharma, Boehringer Ingelheim Pharma GmbH and Co. KG, Cephalon GmbH, Chugai Pharma Marketing Ltd., GBG Forschungs-GmbH, Genentech, Genomic Health, GlaxoSmithKline GmbH and Co. KG, Johnson and Johnson GmbH, Medac mbH, MediGene AG, Mentor $\mathrm{GmbH}$, MSD Sharp and Dohme $\mathrm{GmbH}$, Mundipharma mbH and Co. KG, Novartis Pharma GmbH, Pfizer Pharma GmbH, Pierre Fabre Pharma GmbH, Roche Pharma AG, Sanofi-Aventis GmbH, Siemens AG. The Committee did not consider any of the reported support to represent a conflict of interest that would preclude participation in AGO Breast Committee discussions or voting.

\section{References}

References are available as online supplemental material. To access the references please refer to $w w w$. karger.com/DOI=000331459. 